Cite this: Org. Biomol. Chem., 2013, 11, 6502

Received 9th July 2013,

Accepted 15th August 2013

DOI: $10.1039 /$ c3ob41415a

www.rsc.org/obc

\section{Alkaloid inspired spirocyclic oxindoles from 1,3-dipolar cycloaddition of pyridinium ylides $†$}

\author{
Jonathan Day, ${ }^{a}$ Maliha Uroos, ${ }^{a}$ Richard A. Castledine, ${ }^{a}$ William Lewis, ${ }^{a}$ \\ Ben McKeever-Abbas ${ }^{\mathrm{b}}$ and James Dowden*a
}

Cycloaddition reactions between pyridinium ylides and 3-alkenyl oxindoles that proceed in high yield and with very good regio- and diastereoselectivity are reported. The resulting cycloadducts have the same stereochemistry of biologically active oxindole alkaloids, such as strychnofoline.

\section{Introduction}

Natural products and their signature fragments are an enduring resource for identifying biological modulators. ${ }^{1} \mathrm{~A}$ number of biologically active alkaloids, such as strychnofoline and isorhynchophylline (Fig. 1), feature spiroindolizidine oxindoles. ${ }^{2,3}$ This fragment is considered 'privileged' for potential therapeutic investigation and there is much interest in developing expedient synthesis of such structures. ${ }^{4}$

Target spiroindolizidineoxindole structures may be efficiently accessed by 1,3-dipolar cycloaddition of 3-alkylideneindoline-2-ones. ${ }^{5}$ Serov et al. described reactions between $N$-phenylacylquinolinium ylides with 3 -alkylidene oxindoles, ${ }^{6}$ while extended studies of this type of reaction have recently been reported. ${ }^{7}$ The latter cycloadducts have a relatively high molecular weight due to additional aromatic rings and it is not easy to envisage strategies for their transformation into natural products or drug-like scaffolds.

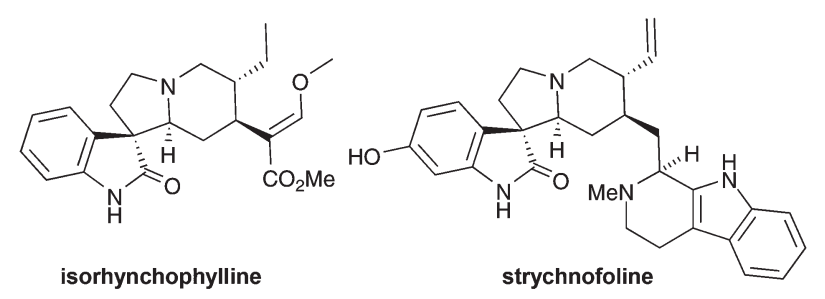

Fig. 1 Example spirooxindole alkaloids.

\footnotetext{
${ }^{a}$ School of Chemistry, University of Nottingham, University Park, Nottingham, NG7 2RD, UK. E-mail: james.dowden@nottingham.ac.uk; Fax: +44 (0)115 9513565; Tel: +44 (0)115 9513566

${ }^{b}$ Pharmaceutical Development, AstraZeneca, Silk Road Business Park, Macclesfield, $U K$

$\dagger$ Electronic supplementary information (ESI) available: Full experimental and spectroscopic information. CCDC 927102-927103. For ESI and crystallographic data in CIF or other electronic format see DOI: 10.1039/c3ob41415a
}

There are no literature descriptions of corresponding 1,3dipolar cycloadditions of pyridinium ylides to 3-alkylidene oxindoles, yet such cycloadducts would be attractive for access to spirooxindole alkaloids and possible therapeutics. Cycloaddition reactions of pyridinium ylides have previously been reported but in situ oxidation is commonly used, leading to valuable unsaturated indolizines. ${ }^{8}$ Of course, such oxidations destroy the rich stereochemical information accumulated during the cycloaddition. Early investigation of general pyridinium ylide cycloadditions noted limited stability of the tetrahydroindolizine cycloadducts and this may have discouraged further investigation of these products. ${ }^{9}$ The 1,2-dihydropyridine motif embedded within tetrahydroindolizine cycloadducts is generally regarded as unstable with few exceptions, ${ }^{10}$ although there have been exciting developments in unlocking their synthetic potential. ${ }^{11}$

This report demonstrates that cycloaddition reactions between pyridinium ylides 2 (Table 1) and 3-alkylidene oxindoles

Table 1 Optimisation of conditions
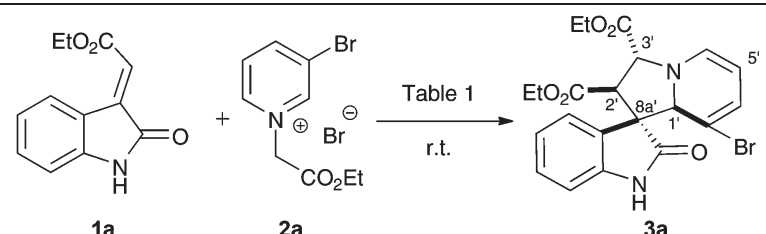

\begin{tabular}{llll}
\hline Solvent & Base & Time (h) & Yield $^{a}(\%)$ \\
\hline Toluene & $\mathrm{Et}_{3} \mathrm{~N}$ & 3 & 65 \\
EtOAc & $\mathrm{Et}_{3} \mathrm{~N}$ & 4 & 64 \\
$\mathrm{CH}_{2} \mathrm{Cl}_{2}$ & $\mathrm{Et}_{3} \mathrm{~N}$ & 2 & 88 \\
$\mathrm{DMF}^{\mathrm{E}}$ & $\mathrm{Et}_{3} \mathrm{~N}$ & 1 & 61 \\
$\mathrm{CH}_{2} \mathrm{Cl}_{2}$ & $\mathrm{Et}_{3} \mathrm{~N}$ & 4 & 40 \\
$\mathrm{CH}_{2} \mathrm{Cl}_{2}$ & $\mathrm{DBU}$ & 3 & 73 \\
$\mathrm{THF}^{t}$ & ${ }^{\mathrm{BuOK}}$ & 3 & 71 \\
& $\mathrm{NaH}$ & 2 & 82
\end{tabular}

${ }^{a}$ Yield after chromatography. 
1 proceed with good selectivity to produce generally isolable spirotetrahydroindolizineoxindole cycloadducts 3 in good yield.

\section{Results and discussion}

Starting materials for this route are readily accessible by olefination of isatin to give 3 -alkylidene-2-oxindoles; ${ }^{12} \dagger$ pyridinium salts were obtained by alkylation. ${ }^{13} \dagger$ Pyridinium salts featuring $N$-methylene groups attached to an electron withdrawing group can be readily deprotonated using mild base to give the ylide. ${ }^{14,15}$

Initially, we set out to briefly investigate the role of solvent and base on yield. An excess of pyridinium salt 2 a relative to dipolarophiles 1a was used to suppress further cycloaddition onto the initial tetrahydroindolizine product. $^{9}$

Generally, high yields of cycloadduct were obtained using a variety of solvents and base at room temperature, although suspending the reagents in dichloromethane, then initiating the reaction by addition of triethylamine is convenient (Table 1). The cycloadduct $\mathbf{3 a}$ is stable to chromatography and could be stored under argon in the freezer for no more than a week.

Cycloaddition between the ylide derived from 3-bromopyridinium salt $\mathbf{2 a}$ and oxindole 1a appears to be highly regioand diastereoselective, giving a single product 3a (Table 1). ${ }^{1} \mathrm{H}-\mathrm{NMR}$ spectroscopy of the cycloadduct revealed an apparent triplet for the $5^{\prime}$ proton $(J=7.0 \mathrm{~Hz})$ indicative of reaction at the C-2 position of the pyridinium salt. Initial NOESY analysis revealed enhancements between protons corresponding to $1^{\prime}$ ring junction and $2^{\prime}$ position adjacent to the spirocentre, but not between the $1^{\prime}$ and $3^{\prime}$ positions adjacent to nitrogen.

Ultimately, single crystal X-ray diffraction of this material gave unequivocal evidence of the diastereoselectivity of the reaction (Fig. 2). $\neq$ The relative stereochemistry of the spirocentre (position $8 \mathrm{a}^{\prime}$ ) and the $1^{\prime}$ position is diagnostic of exo or endo selectivity; co-location of the oxindole carbonyl and $1^{\prime}$ proton on the same face of the cycloadduct is suggestive of an attractive interaction between the electron rich oxindole aromatic ring and the electron deficient pyridinium in the transition state. The trans arrangement of the $1^{\prime}$ and $3^{\prime}$ protons is also confirmed, indicating that the ylide is $S$-shaped in the transition state (Scheme 1). This relative stereochemistry is the same as that required for alkaloids related to strychnofoline and isorhynchophylline.

We next carried out investigation into the generality of the reaction using various 3 -alkylideneoxindoles $\mathbf{1 b}-\mathbf{f}$ (Table 2). ${ }^{11} \dagger$ Cycloaddition with the ylide derived from 3-bromopyridinium

$\neq$ Crystal data for 3a. $\mathrm{C}_{21} \mathrm{H}_{21} \mathrm{BrN}_{2} \mathrm{O}_{5}, M=507.38$, triclinic, $a=8.5537(12), b=$ 9.0560(14), $c=16.2097(17) \AA, U=1147.4(3) \AA^{3} . T=120(2) \mathrm{K}$, space group $P \overline{1}, Z=$ 2, 8522 reflections measured, 4490 unique with $R=0.0804, \mathrm{w} R_{2}=0.2241$. CCDC 927103. Crystal data for $3 \mathbf{g}^{\prime} \cdot \mathrm{C}_{19} \mathrm{H}_{17} \mathrm{~N}_{3} \mathrm{O}_{3}, M=335.35$, triclinic, $a=8.8865(5), b=$ 9.3125(3), $c=10.9460(5) \AA, U=826.26(7) \AA^{3} . T=120(2) \mathrm{K}$, space group $P \overline{1}, Z=2$, 15441 reflections measured, 3480 unique with $R=0.0346, \mathrm{w} R_{2}=0.0903$. CCDC 927102.

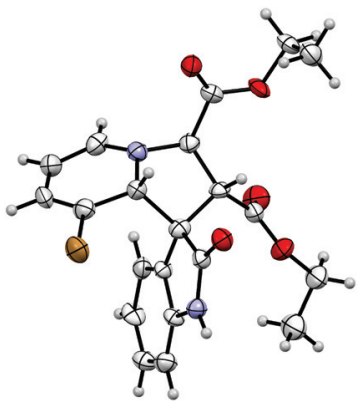

Fig. 2 Crystal structure of cycloadduct 3a (CCDC 927103). Ellipsoids are drawn at the $50 \%$ probability level.

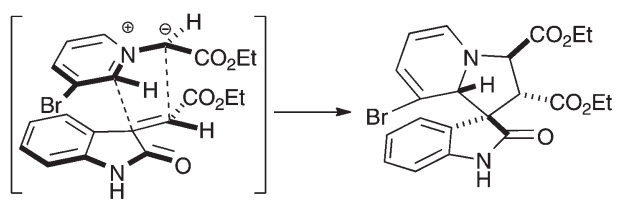

Scheme 1 Proposed origin of observed diastereoselectivity.

salt 2a generally proceeded to give good yields (80-90\%) of cycloadducts $\mathbf{3 b}-\mathbf{f}$ as single diastereoisomers with relative stereochemistry similar to cycloadduct $3 \mathbf{a}$ as judged by ${ }^{1} \mathrm{H}$ NMR spectroscopy. 3-Methylene oxindole 1f is a stable solid that readily undergoes cycloaddition reactions in good yield (entry 5). Unfortunately, $\mathrm{N}$-substituted 3-methylene oxindoles are highly reactive and generally met with polymerisation before cycloaddition could be attempted. ${ }^{16}$ On the other hand, $N$-propargyl and $N$-acetyl substituted oxindoles (entries 3 and 4, 1d and 1e respectively) bearing an ethyl ester gave good yields of cycloadducts ( $3 \mathbf{d}$ and $3 \mathbf{e}$ respectively).

A selection of pyridinium salts $(2 \mathbf{g}-\mathbf{l})$ were also evaluated (Table 3). Cycloadducts arising from reaction with unsubstituted pyridinium salt (2l) could be detected by mass spectrometry of the reaction mixture but were not stable to purification by chromatography, however the saturated indolizidine 31 could be obtained by in situ reduction using RANEY® nickel in good yield.

Pyridinium salts featuring methyl, or phenyl substituents at the 3- or 4-pyridinium position (not shown) did not give observable products in their respective cycloaddition reactions using these condition, which we presume to be due to their instability.

Pleasingly, less acidic pyridinium salts featuring either nitrile (2m) or phenyl (2n) ylide stabilising groups could be deprotonated with sodium hydride, leading to good yields of the corresponding cycloadducts $\mathbf{3 m}$ and $\mathbf{3 n}$ respectively as single diastereoisomers (Scheme 2).

Pyridinium salts featuring resonance stabilising groups $(\mathbf{2 g}-\mathbf{i})$ in the 3-position gave good overall yields of spiroindolizidine oxindoles arising from reaction at the C- 6 position of the pyridinium ring, i.e. opposite to the electron withdrawing substituent. These products $(\mathbf{3 g}-\mathbf{i})$ were obtained as a mixture of two diastereoisomers in varying ratios. 
Table 2 Cycloaddition with varied oxindoles

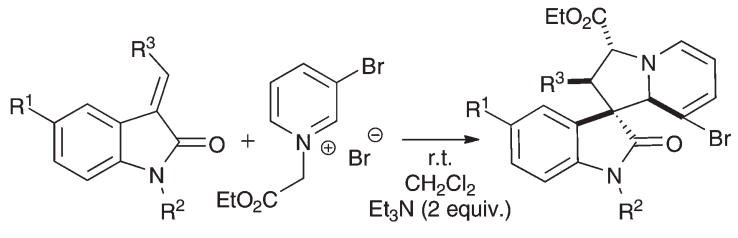

1- 1 equiv. $\quad 2 a-2$ equiv.

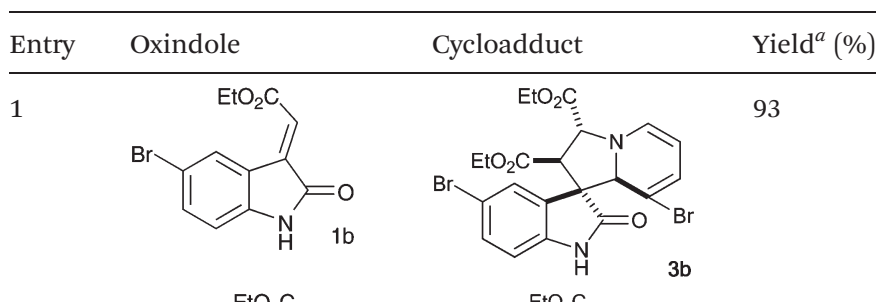

2<smiles>CCOC(=O)/C=C1/C(=O)Nc2ccc([N+](=O)[O-])cc21</smiles>

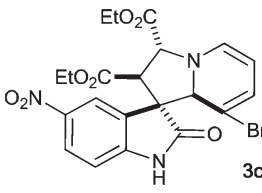

91<smiles>C#CCN1C(=O)/C(=C/C(=O)OCC)c2cc(OC)ccc21</smiles>

4<smiles>CCOC(=O)C=C1C(=O)N(C(C)=O)c2ccccc21</smiles>

5<smiles>C=C1C(=O)Nc2ccccc21</smiles>

${ }^{a}$ Yield after chromatography.

Crystals were obtained from the 3-cyanopyridine derived cycloadduct $3 \mathrm{~g}$ that X-ray diffraction revealed to feature the $1^{\prime}$ proton on the opposite face to the oxindole carbonyl (Fig. 3). $\neq$ This product was later confirmed as the minor diastereoisomer $3 \mathbf{g}^{\prime}$ by ${ }^{1} \mathrm{H}$ NMR spectroscopy.

There are two possible explanations for these observed results. One is that pyridinium resonance stabilising groups influence the orientation of the transition state. Alternatively, interconversion of cycloadducts via ring opening to restore the pyridinium ring, then rotation about the former 2 '-8'a bond and rejoining of the enolate with the pyridinium may proceed. ${ }^{17}$ An analogous reversible Mannich reaction mechanism is well known to occur in either acidic or basic media, leading to interchange between related spirocyclic oxindole alkaloid diastereoisomers, such as isorhynchophylline and
Table 3 Cycloaddition with various pyridinium salts

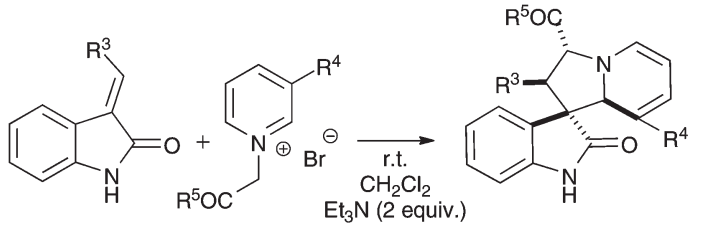

1a/1f - 1 equiv. 2 - 2 equiv. 3<smiles>CCOC(=O)C[C@@H]1C[C@@]2(C(=O)Nc3ccccc32)[C@@H]2C=CC(C#N)=CN12</smiles>

2
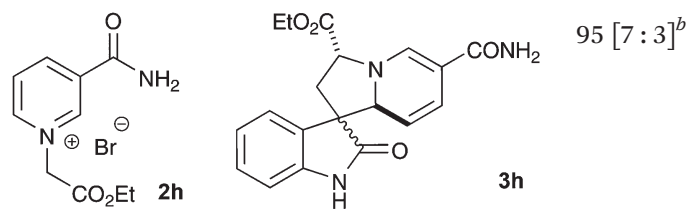

3<smiles>CCOC(=O)C[n+]1cccc(C(C)=O)c1</smiles><smiles>CCOC(=O)[C@@H]1C[C@]2(C(=O)Nc3ccccc32)[C@@H]2C=CC(C(N)=O)=CN12</smiles>

4<smiles>CC(=O)C[n+]1cccc(Br)c1</smiles><smiles>CCOC(=O)Nc1ccccc1[C@]12C[C@@H](OC)N3C=CC=C(Br)[C@H]3[C@H]1C2=O</smiles>

5<smiles>CCOC(=O)C[n+]1cccc(F)c1</smiles><smiles>CCOC(=O)C1[C@H](C(=O)OCC)[C@@]2(C(=O)Nc3ccccc32)C2C(F)=CC=CN12</smiles>

6<smiles>CCOC(=O)C[n+]1ccccc1Br</smiles>

$81[1: 1]^{b}$

i

76

87

$63^{c}$

${ }^{a}$ Yield after chromatography. ${ }^{b}$ Inseparable mixture of two diastereoisomers, ratio in parenthesis from relative integration of ${ }^{1} \mathrm{H}$ NMR spectrum. ${ }^{c}$ Crude cycloadduct subject to $\mathrm{H}_{2} / \mathrm{RANEY}{ }^{\circledR}$ nickel $(\sim 10 \mathrm{~mol} \%)$ in EtOAc.

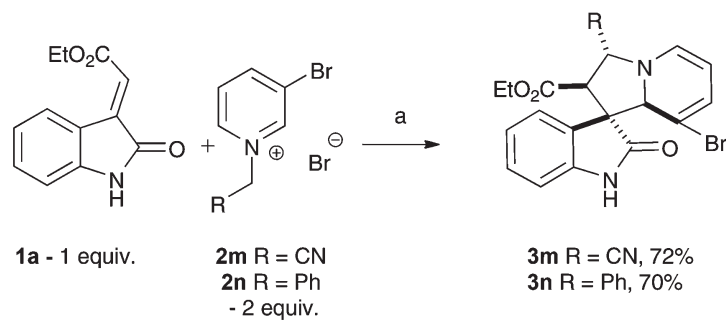

Scheme 2 Reagents and conditions: a, NaH (2 equiv.), THF, rt. 


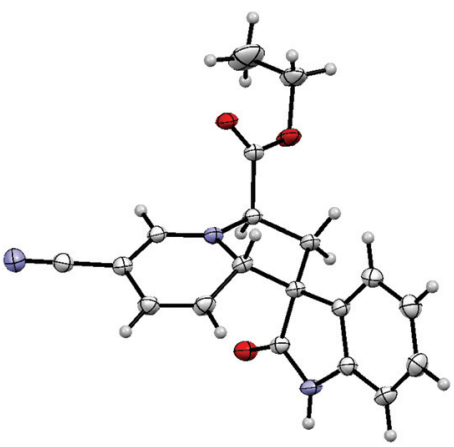

Fig. 3 Crystal structure of the minor diastereoisomer of cycloadduct $\mathbf{3} \mathbf{g}^{\prime}$ (CCDC 927102). Ellipsoids are drawn at the 50\% probability level.

rhynchophylline. ${ }^{18}$ Diastereoisomer $3 \mathbf{g}^{\prime}$ remained unchanged when exposed to the same cycloaddition conditions for 14 hours, however. ${ }^{19}$

A recent paper described reactions of very closely related pyridinium ylides and 3-alkylideneoxindoles in ethanol with $20 \mathrm{~mol} \%$ triethylamine at $50{ }^{\circ} \mathrm{C}$, but alkene products arising from elimination of pyridine were the exclusive reported products. ${ }^{20}$ A similar reaction product was obtained when the cycloaddition reaction between pyridinium ylide $2 \mathbf{l}$ with 3-alkylideneoxindole 1a was performed in ethanol at room temperature, resulting in clean conversion to the elimination product 5 in $60 \%$ yield (Scheme 3 ). Presumably, the reaction proceeds via 1,4-conjugate addition and not cycloaddition, with subsequent elimination of pyridine. ${ }^{21,22}$

Significantly, crude cycloadduct 4 obtained from cycloaddition in dichloromethane at room temperature did not undergo elimination when stirred in ethanol with triethylamine. This result, when considered alongside the observation for cycloadduct $\mathbf{3} \mathbf{g}^{\prime}$ above, suggests that the reversible mechanism is not occurring in these cases. Overall, these studies underline that, for these reagents, cycloaddition and 1,4-conjugate addition reaction outcomes are under the subtle influence of solvent.

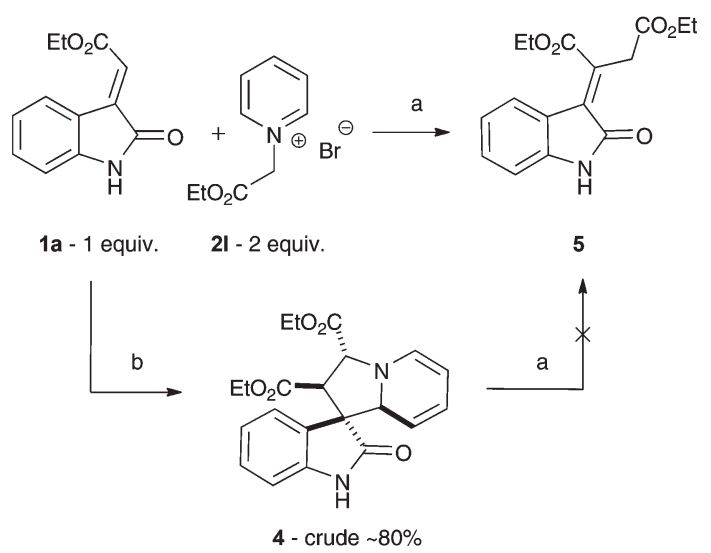

Scheme 3 Reagents and conditions: $\mathrm{a}_{1} \mathrm{Et}_{3} \mathrm{~N}$ (2 equiv.), ethanol, $\mathrm{rt}, 60 \% ; \mathrm{b}, \mathrm{Et}_{3} \mathrm{~N}$ (2 equiv.), $\mathrm{CH}_{2} \mathrm{Cl}_{2}$, rt.

\section{Conclusions}

In summary, 1,3-dipolar cycloadditions between pyridinium ylides and 3-alkenyloxindoles that give spiroindolizidine oxindole products in high yield and with excellent regioselectivity and diastereoselectivity are reported for the first time. These cycloadducts are highly reminiscent of biologically active alkaloids, especially isorhynchophylline and strychnofoline. Specific modification of this general cycloaddition route to facilitate highly convergent access to such alkaloids and related targets is underway.

\section{Experimental}

\section{General information}

Experimental describing precursor preparation is included within the ESI. $\uparrow$

General procedure for preparation of cycloadducts (3). Base ( 2 equiv.) was added to pyridinium salt (2 equiv.) in dry solvent, under argon and the mixture stirred at room temperature for 5 minutes. Oxindole ( 1 equiv.) was added and the mixture stirred at room temperature for 2-4 hours. Water was added and the organic layer separated, washed with water $(2 \times)$, brine, dried over $\mathrm{Na}_{2} \mathrm{SO}_{4}$, filtered and evaporated. The crude products were purified by column chromatography on silica gel (using either $\mathrm{MeOH}-\mathrm{CH}_{2} \mathrm{Cl}_{2} ; 1 / 10$ or EtOAc-petrol; 1/1 as eluent) or recrystallised with ethanol.

$\left(1^{\prime} R^{*}, 2^{\prime} S^{*}, 3^{\prime} S^{*}, 8 a^{\prime} S^{*}\right)$-Diethyl $\quad 8^{\prime}$-bromo-2-oxo-3',8a'-dihydro$\mathbf{2}^{\prime} \boldsymbol{H}$-spiro[indoline-3,1'-indolizine]-2',3'-dicarboxylate (3a). Brown solid; $121 \mathrm{mg}$ (88\%); m.p. $76-78{ }^{\circ} \mathrm{C}$; IR $\left(\nu_{\max } / \mathrm{cm}^{-1}, \mathrm{CHCl}_{3}\right)$ 3442, 3136, 2984, 2482, 1734, 1639, 1622, 1563, 1471, 1372, 1340, 1249, 1191; NMR $\delta_{\mathrm{H}}\left(400 \mathrm{MHz}, d^{6}\right.$-DMSO) $10.68(1 \mathrm{H}, \mathrm{br}$ s, NH), 7.26-7.22 (1H, m, ArH), $7.17(1 \mathrm{H}, \mathrm{d}, J=7.4 \mathrm{~Hz}, \operatorname{Ar} H)$, $6.91(1 \mathrm{H}, \mathrm{m}, \operatorname{Ar} H), 6.86(1 \mathrm{H}, \mathrm{d}, J=7.4 \mathrm{~Hz}, \operatorname{Ar} H), 6.61(1 \mathrm{H}, \mathrm{d}, J=$ $7.0 \mathrm{~Hz}, \mathrm{~N}-\mathrm{CH}=\mathrm{CH}), 6.10(1 \mathrm{H}, \mathrm{d}, J=7.0 \mathrm{~Hz}, \mathrm{CH}-\mathrm{C} H=\mathrm{CBr})$, $5.06\left(1 \mathrm{H}, \mathrm{d}, J=7.0 \mathrm{~Hz}, \mathrm{NCH}\left(\mathrm{CO}_{2} \mathrm{Et}\right), 5.00\right.$ (1H, s, NCHC-Br), $4.45(1 \mathrm{H}$, app t $, J=7.0 \mathrm{~Hz}, \mathrm{NCH}=\mathrm{CH}), 4.21(2 \mathrm{H}, \mathrm{q}, J=7.1 \mathrm{~Hz}$, $\left.\mathrm{NCHCO}_{2} \mathrm{CH}_{2} \mathrm{CH}_{3}\right), 3.65\left(2 \mathrm{H}, \mathrm{q}, J=7.1 \mathrm{~Hz}, \mathrm{CCH}\left(\mathrm{CO}_{2} \mathrm{CH}_{2} \mathrm{CH}_{3}\right)\right.$ ), $3.45\left(1 \mathrm{H}, \mathrm{d}, J=7.0 \mathrm{~Hz}, \mathrm{CCH}\left(\mathrm{CO}_{2} \mathrm{Et}\right), 1.23(3 \mathrm{H}, \mathrm{t}, J=7.1 \mathrm{~Hz}\right.$, $\left.\mathrm{NCHCOCH}_{2} \mathrm{CH}_{3}\right), 0.62\left(3 \mathrm{H}, \mathrm{t}, J=7.1 \mathrm{~Hz}, \mathrm{CCO}_{2} \mathrm{CH}_{2} \mathrm{CH}_{3}\right)$; NMR $\delta_{\mathrm{C}}\left(100 \mathrm{MHz}, d^{6}\right.$-DMSO) $176.2(\mathrm{C}), 171.4(\mathrm{C}), 168.4(\mathrm{C}), 144.0$ (C), $134.9(\mathrm{CH}), 129.5(\mathrm{CH}), 128.9(\mathrm{CH}), 126.5(\mathrm{C}), 124.7(\mathrm{CH})$, $121.5(\mathrm{CH}), 109.9(\mathrm{CH}), 106.9(\mathrm{C}), 93.2(\mathrm{CH}), 72.6(\mathrm{CH}), 64.8(\mathrm{CH})$, $64.2(\mathrm{C}), 62.0\left(\mathrm{CH}_{2}\right), 61.4\left(\mathrm{CH}_{2}\right), 52.8(\mathrm{CH}), 14.5\left(\mathrm{CH}_{3}\right), 13.5\left(\mathrm{CH}_{3}\right)$; $m / z$ (HRMS-ESI + ) $461.0697\left(\mathrm{M}+\mathrm{H} \mathrm{C}_{21} \mathrm{H}_{22}{ }^{79} \mathrm{BrN}_{2} \mathrm{O}_{5}\right.$ requires 461.0712), $463.0700\left(\mathrm{M}+\mathrm{H} \mathrm{C}_{21} \mathrm{H}_{22}{ }^{81} \mathrm{BrN}_{2} \mathrm{O}_{5}\right.$ requires 463.0692).

$\left(1^{\prime} R^{*}, 2^{\prime} S^{*}, 3^{\prime} S^{*}, 8 a^{\prime} S^{*}\right)$-Diethyl $5,8^{\prime}$-dibromo-2-oxo-3',8a'-dihydro$\mathbf{2}^{\prime} \boldsymbol{H}$-spiro[indoline-3,1'-indolizine]-2',3'-dicarboxylate (3b). Brown solid; $85 \mathrm{mg}$ (93\%); m.p. $68-70{ }^{\circ} \mathrm{C}$; IR $\left(\nu_{\max } / \mathrm{cm}^{-1}, \mathrm{CHCl}_{3}\right)$ 3437, 2982, 1732, 1639, 1618, 1564, 1465, 1373, 1309, 1130; NMR $\delta_{\mathrm{H}}\left(400 \mathrm{MHz}, d^{6}\right.$-acetone) $9.84(1 \mathrm{H}$, br s, NH), 7.45-7.41 $(2 \mathrm{H}, \mathrm{m}, \mathrm{Ar} H), 6.95$ (1H, dd $J=8.2,0.4 \mathrm{~Hz}, \operatorname{Ar} H), 6.61(1 \mathrm{H}, \mathrm{d}$, $J=7.1 \mathrm{~Hz}, \mathrm{~N}-\mathrm{CH}=\mathrm{CH}), 6.14(1 \mathrm{H}$, app dt, $J=6.4,1.0 \mathrm{~Hz}, \mathrm{CH}-$ $\mathrm{CH}=\mathrm{CBr}), 5.20(1 \mathrm{H}, \mathrm{d}, J=1.0 \mathrm{~Hz}, \mathrm{NCHCBr}), 5.05(1 \mathrm{H}, \mathrm{d}, J=6.6$ $\mathrm{Hz}, \mathrm{NCH}\left(\mathrm{CO}_{2} \mathrm{Et}\right), 4.58(1 \mathrm{H}, \mathrm{dd}, J=7.1,6.4 \mathrm{~Hz}, \mathrm{NCH}=\mathrm{CH}), 4.27$ 
$\left(2 \mathrm{H}, \mathrm{q}, J=7.1 \mathrm{~Hz}, \mathrm{NCHCO}_{2} \mathrm{CH}_{2} \mathrm{CH}_{3}\right), 3.76(2 \mathrm{H}, \mathrm{q}, J=7.1 \mathrm{~Hz}$, $\left.\mathrm{CCHCO}_{2} \mathrm{CH}_{2} \mathrm{CH}_{3}\right), 3.69\left(1 \mathrm{H}, \mathrm{d}, J=6.6 \mathrm{~Hz}, \mathrm{CCHCO}_{2} \mathrm{Et}\right), 1.29$ $\left(3 \mathrm{H}, \mathrm{t}, J=7.1 \mathrm{~Hz}, \mathrm{NCHCOCH}_{2} \mathrm{CH}_{3}\right), 0.78(3 \mathrm{H}, \mathrm{t}, J=7.1 \mathrm{~Hz}$, $\left.\mathrm{CCHCO}_{2} \mathrm{CH}_{2} \mathrm{CH}_{3}\right)$; NMR $\delta_{\mathrm{C}}\left(100 \mathrm{MHz}, d^{6}\right.$-acetone) $175.3(\mathrm{C})$, 170.6 (C), 168.0 (C), 143.0 (C), $134.0(\mathrm{CH}), 131.9(\mathrm{CH}), 129.0$ (C), $128.8(\mathrm{CH}), 127.5(\mathrm{CH}), 113.3(\mathrm{C}), 111.3(\mathrm{CH}), 106.8(\mathrm{C})$, $93.3(\mathrm{CH}), 72.8(\mathrm{CH}), 64.8(\mathrm{CH}), 64.4(\mathrm{C}), 61.6\left(\mathrm{CH}_{2}\right), 61.1$ $\left(\mathrm{CH}_{2}\right), 52.8(\mathrm{CH}), 13.6\left(\mathrm{CH}_{3}\right), 12.9\left(\mathrm{CH}_{3}\right) ; \mathrm{m} / \mathrm{z}$ (HRMS-ESI+) $538.9808\left(\mathrm{M}+\mathrm{H} \mathrm{C}_{21} \mathrm{H}_{21}{ }^{79} \mathrm{Br}_{2} \mathrm{~N}_{2} \mathrm{O}_{5}\right.$ requires 538.9812), 540.9806 $\left(\mathrm{M}+\mathrm{H} \mathrm{C}_{21} \mathrm{H}_{21}{ }^{81} \mathrm{Br}_{2} \mathrm{~N}_{2} \mathrm{O}_{5}\right.$ requires 540.9792).

$\left(1^{\prime} R^{*}, 2^{\prime} S^{*}, 3^{\prime} S^{*}, 8 a^{\prime} S^{*}\right)$-Diethyl 8'-bromo-5-nitro-2-oxo-3',8a'dihydro-2' $\boldsymbol{H}$-spiro[indoline-3,1'-indolizine]-2', $\mathbf{3}^{\prime}$-dicarboxylate (3c). Yellow solid; $88 \mathrm{mg}$ (91\%); m.p. $83-85^{\circ} \mathrm{C}$; IR $\left(\nu_{\max } / \mathrm{cm}^{-1}\right.$, $\left.\mathrm{CHCl}_{3}\right) 3249,2981,1745,1626,1603,1455,1343,1125 ; \mathrm{NMR} \delta_{\mathrm{H}}$ (400 MHz, $d^{6}$-acetone) $10.30(1 \mathrm{H}, \mathrm{br} \mathrm{s}, \mathrm{NH}), 8.28(1 \mathrm{H}, \mathrm{dd}, J=8.7$, $2.3 \mathrm{~Hz}, \operatorname{Ar} H), 8.17(1 \mathrm{H}, \mathrm{d}, J=2.3 \mathrm{~Hz}, \operatorname{Ar} H), 7.22(1 \mathrm{H}, \mathrm{d}, J=8.7$ $\mathrm{Hz}, \operatorname{Ar} H), 6.70(1 \mathrm{H}, \mathrm{d}, J=7.1 \mathrm{~Hz}, \mathrm{~N}-\mathrm{CH}=\mathrm{CH}), 6.15(1 \mathrm{H}, \mathrm{d}, J=$ $6.4 \mathrm{~Hz}, \mathrm{CH}-\mathrm{CH}=\mathrm{CBr}), 5.27(1 \mathrm{H}, \mathrm{s}, \mathrm{NCHC}-\mathrm{Br}), 5.15(1 \mathrm{H}, \mathrm{d}, J=$ $\left.6.5 \mathrm{~Hz}, \mathrm{NCH}\left(\mathrm{CO}_{2} \mathrm{Et}\right)\right), 4.62(1 \mathrm{H}, \mathrm{dd}, J=7.1,6.4 \mathrm{~Hz}, \mathrm{NCH}=\mathrm{CH})$, $4.28\left(2 \mathrm{H}, \mathrm{q}, J=7.1 \mathrm{~Hz}, \mathrm{NCHCO}_{2} \mathrm{CH}_{2} \mathrm{CH}_{3}\right), 3.77(1 \mathrm{H}, \mathrm{d}, J=6.5$ $\left.\mathrm{Hz}, \mathrm{CCH}\left(\mathrm{CO}_{2} \mathrm{Et}\right)\right), 3.76\left(2 \mathrm{H}, \mathrm{q}, J=7.1 \mathrm{~Hz}, \mathrm{CCH}\left(\mathrm{CO}_{2} \mathrm{CH}_{2} \mathrm{CH}_{3}\right)\right)$, $1.30\left(3 \mathrm{H}, \mathrm{t}, J=7.1 \mathrm{~Hz}, \mathrm{NCHCOCH}_{2} \mathrm{CH}_{3}\right), 0.77(3 \mathrm{H}, \mathrm{t}, J=7.1 \mathrm{~Hz}$, $\left.\mathrm{CCH}\left(\mathrm{CO}_{2} \mathrm{CH}_{2} \mathrm{CH}_{3}\right)\right)$; NMR $\delta_{\mathrm{C}}\left(100 \mathrm{MHz}, d^{6}\right.$-acetone) $176.0(\mathrm{C})$, 170.4 (C), 167.9 (C), 149.9 (C), $142.4(\mathrm{C}), 134.0(\mathrm{CH}), 129.1(\mathrm{CH})$, $127.5(\mathrm{C}), 126.3(\mathrm{CH}), 120.2(\mathrm{CH}), 109.7(\mathrm{CH}), 106.4(\mathrm{C}), 93.6$ $(\mathrm{CH}), 73.2(\mathrm{CH}), 65.0(\mathrm{CH}), 64.2(\mathrm{C}), 61.7\left(\mathrm{CH}_{2}\right), 61.4\left(\mathrm{CH}_{2}\right), 52.8$ $(\mathrm{CH}), 13.6\left(\mathrm{CH}_{3}\right), 12.9\left(\mathrm{CH}_{3}\right) ; \mathrm{m} / z$ (HRMS-ESI +$) 506.0569(\mathrm{M}+\mathrm{H}$ $\mathrm{C}_{21} \mathrm{H}_{21}{ }^{79} \mathrm{BrN}_{3} \mathrm{O}_{7}$ requires 506.0558), $508.0552\left(\mathrm{M}+\mathrm{H} \mathrm{C}_{21} \mathrm{H}_{21}\right.$ ${ }^{81} \mathrm{BrN}_{3} \mathrm{O}_{7}$ requires 508.0537).

$\left(1^{\prime} R^{*}, 2^{\prime} S^{*}, 3^{\prime} S^{*}, 8 a^{\prime} S^{*}\right)$-Diethyl $\quad 8^{\prime}$-bromo-5-methoxy-2-oxo-1(prop-2-yn-1-yl)-3',8a'-dihydro-2' $H$-spiro[indoline-3,1'-indolizine]-2',3'-dicarboxylate (3d). Grey solid; $74 \mathrm{mg}$ (82\%); m.p. $125-127{ }^{\circ} \mathrm{C}$ (ethanol); IR $\left(\nu_{\max } / \mathrm{cm}^{-1}, \mathrm{CHCl}_{3}\right) 3309,3011$, $1738,1718,1639,1605,1495,1370,1339,1192 ; \mathrm{NMR} \delta_{\mathrm{H}}$ (400 MHz, d $d^{6}$-acetone) $7.07(1 \mathrm{H}, \mathrm{d}, J=8.5 \mathrm{~Hz}, \mathrm{Ar} H), 6.99(1 \mathrm{H}$, $\mathrm{d}, J=2.4 \mathrm{~Hz}, \operatorname{Ar} H), 6.94(1 \mathrm{H}, \mathrm{dd}, J=8.5,2.4 \mathrm{~Hz}, \operatorname{Ar} H), 6.61(1 \mathrm{H}$, $\mathrm{d}, J=7.1 \mathrm{~Hz}, \mathrm{~N}-\mathrm{CH}=\mathrm{CH}), 6.08(1 \mathrm{H}, \mathrm{dt}, J=6.4,0.9 \mathrm{~Hz}, \mathrm{CH}-$ $\mathrm{CH}=\mathrm{CBr}), 5.21(1 \mathrm{H}, \mathrm{d}, J=0.9 \mathrm{~Hz}, \mathrm{NCHC}-\mathrm{Br}), 5.06(1 \mathrm{H}, \mathrm{d}, J=$ $\left.6.9 \mathrm{~Hz}, \mathrm{NCH}\left(\mathrm{CO}_{2} \mathrm{Et}\right)\right), 4.63\left(1 \mathrm{H}, \mathrm{d}, J=17.8,2.6 \mathrm{~Hz}, \mathrm{NCH}_{\mathrm{a}} \mathrm{H}_{\mathrm{b}}\right)$, $4.57(1 \mathrm{H}, \mathrm{dd}, J=7.1,6.4 \mathrm{~Hz}, \mathrm{NCH}=\mathrm{CH}), 4.50(1 \mathrm{H}, \mathrm{d}, J=17.8$, $\left.2.5 \mathrm{~Hz}, \mathrm{NCH}_{\mathrm{a}} H_{\mathrm{b}}\right), 4.27\left(2 \mathrm{H}, \mathrm{q}, J=7.1 \mathrm{~Hz}, \mathrm{NCHCO}_{2} \mathrm{CH}_{2} \mathrm{CH}_{3}\right)$, $3.74\left(3 \mathrm{H}, \mathrm{s}, \mathrm{OCH}_{3}\right), 3.73\left(1 \mathrm{H}, \mathrm{d}, J=6.9 \mathrm{~Hz}, \mathrm{CCH}\left(\mathrm{CO}_{2} \mathrm{Et}\right), 3.72\right.$ $\left(2 \mathrm{H}, \mathrm{q}, J=7.1 \mathrm{~Hz}, \mathrm{CCH}\left(\mathrm{CO}_{2} \mathrm{CH}_{2} \mathrm{CH}_{3}\right)\right), 2.80(1 \mathrm{H}, \mathrm{dd}, J=2.6,2.5$ $\mathrm{Hz}, \mathrm{C} \equiv \mathrm{CH}), 1.30\left(3 \mathrm{H}, \mathrm{t}, J=7.1 \mathrm{~Hz}, \mathrm{NCHCOCH}_{2} \mathrm{CH}_{3}\right), 0.73$ $\left(3 \mathrm{H}, \mathrm{t}, J=7.1 \mathrm{~Hz}, \mathrm{CCH}\left(\mathrm{CO}_{2} \mathrm{CH}_{2} \mathrm{CH}_{3}\right)\right) ; \mathrm{NMR} \delta_{\mathrm{C}}(100 \mathrm{MHz}$, $d^{6}$-acetone) $172.8(\mathrm{C}), 170.7(\mathrm{C}), 167.9$ (C), 155.6 (C), 137.0 (C), $133.9(\mathrm{CH}), 128.5(\mathrm{CH}), 126.9(\mathrm{C}), 113.3(\mathrm{CH}), 111.8(\mathrm{CH}), 109.3$ $(\mathrm{CH}), 106.4(\mathrm{C}), 93.3(\mathrm{CH}), 76.9(\mathrm{C}), 72.8(\mathrm{CH}), 72.7(\mathrm{C}), 64.8$ $(\mathrm{CH}), 61.5\left(\mathrm{CH}_{2}\right), 61.0\left(\mathrm{CH}_{2}\right), 52.3(\mathrm{CH}), 55.1(\mathrm{CH}), 52.3\left(\mathrm{CH}_{3}\right)$, $29.2\left(\mathrm{CH}_{2}\right), 13.6\left(\mathrm{CH}_{3}\right), 12.9\left(\mathrm{CH}_{3}\right) ; \mathrm{m} / z$ (HRMS-ESI+) 529.0957 $\left(\mathrm{M}+\mathrm{H} \mathrm{C}_{25} \mathrm{H}_{26}{ }^{79} \mathrm{BrN}_{2} \mathrm{O}_{6}\right.$ requires 529.0969), $531.0951(\mathrm{M}+\mathrm{H}$ $\mathrm{C}_{25} \mathrm{H}_{26}{ }^{81} \mathrm{BrN}_{2} \mathrm{O}_{6}$ requires 531.0949).

$\left(1^{\prime} R^{*}, 2^{\prime} S^{*}, 3^{\prime} S^{*}, 8 a^{\prime} S^{*}\right)$-Diethyl 1-acetyl-8'-bromo-2-oxo-3',8a'dihydro-2' $H$-spiro[indoline-3, $\mathbf{1}^{\prime}$-indolizine]-2', $3^{\prime}$-dicarboxylate (3e). Yellow solid; $80 \mathrm{mg}$ (83\%); m.p. 108-110 ${ }^{\circ} \mathrm{C}$; IR $\left(\nu_{\max } /\right.$ $\mathrm{cm}^{-1}, \mathrm{CHCl}_{3}$ ) 2981, 1744, 1710, 1636, 1561, 1466, 1370, 1308,
1272, 1191, 1023; NMR $\delta_{\mathrm{H}}\left(400 \mathrm{MHz}, d^{6}\right.$-acetone) $8.22(1 \mathrm{H}, \mathrm{d}$, $J=8.2 \mathrm{~Hz}, \operatorname{Ar} H), 7.45-7.39(2 \mathrm{H}, \mathrm{m}, \mathrm{ArH}), 7.21(1 \mathrm{H}, \mathrm{td}, J=8.2,1.4$ $\mathrm{Hz}, \mathrm{Ar} H), 6.63(1 \mathrm{H}, \mathrm{d}, J=7.1 \mathrm{~Hz}, \mathrm{~N}-\mathrm{CH}=\mathrm{CH}), 6.11(1 \mathrm{H}, \mathrm{d}, J=$ $6.5 \mathrm{~Hz}, \mathrm{CH}-\mathrm{CH}=\mathrm{CBr}), 5.37(1 \mathrm{H}, \mathrm{s}, \mathrm{NCHC}-\mathrm{Br}), 5.05(1 \mathrm{H}, \mathrm{d}, J=$ $\left.7.3 \mathrm{~Hz}, \mathrm{NCH}\left(\mathrm{CO}_{2} \mathrm{Et}\right)\right), 4.58(1 \mathrm{H}, \mathrm{dd}, J=7.1,6.5 \mathrm{~Hz}, \mathrm{NCH}=\mathrm{CH})$, $4.29\left(2 \mathrm{H}, \mathrm{q}, J=7.1 \mathrm{~Hz}, \mathrm{NCHCO}_{2} \mathrm{CH}_{2} \mathrm{CH}_{3}\right), 3.86(1 \mathrm{H}, \mathrm{d}, J=7.3$ $\mathrm{Hz}, \mathrm{CCH}\left(\mathrm{CO}_{2} \mathrm{Et}\right), 3.69\left(2 \mathrm{H}, \mathrm{q}, J=7.1 \mathrm{~Hz}, \mathrm{CCH}\left(\mathrm{CO}_{2} \mathrm{CH}_{2} \mathrm{CH}_{3}\right)\right)$, $2.66\left(3 \mathrm{H}, \mathrm{s}, \mathrm{NCOCH}_{3}\right), 1.31\left(3 \mathrm{H}, \mathrm{t}, J=7.1 \mathrm{~Hz}, \mathrm{NCHCOCH}_{2} \mathrm{CH}_{3}\right)$, $0.69\left(3 \mathrm{H}, \mathrm{t}, J=7.1 \mathrm{~Hz}, \mathrm{CCH}\left(\mathrm{CO}_{2} \mathrm{CH}_{2} \mathrm{CH}_{3}\right)\right)$; NMR $\delta_{\mathrm{C}}(100 \mathrm{MHz}$, $d^{6}$-acetone) $175.3(\mathrm{C}), 170.4(\mathrm{C}), 170.3$ (C), 167.4 (C), 141.5 (C), $133.8(\mathrm{CH}), 129.4(\mathrm{CH}) .129 .1(\mathrm{CH}), 125.4(\mathrm{C}), 124.7(\mathrm{CH}), 123.9$ $(\mathrm{CH}), 115.9(\mathrm{CH}), 106.3(\mathrm{C}), 93.5(\mathrm{CH}), 74.3(\mathrm{CH}), 64.5(\mathrm{CH}), 63.8$ (C), $61.7\left(\mathrm{CH}_{2}\right), 61.2\left(\mathrm{CH}_{2}\right), 53.6(\mathrm{CH}), 25.8\left(\mathrm{CH}_{3}\right), 13.5\left(\mathrm{CH}_{3}\right)$, $12.7\left(\mathrm{CH}_{3}\right) ; \mathrm{m} / z$ (HRMS-ESI+) $503.0800\left(\mathrm{M}+\mathrm{H} \mathrm{C}_{23} \mathrm{H}_{24}{ }^{79} \mathrm{BrN}_{2} \mathrm{O}_{6}\right.$ requires 503.0813), $505.0788\left(\mathrm{M}+\mathrm{H} \mathrm{C}_{23} \mathrm{H}_{24}{ }^{81} \mathrm{BrN}_{2} \mathrm{O}_{6}\right.$ requires 505.0792).

$\left(1^{\prime} S^{*}, 3^{\prime} R^{*}, 8 a^{\prime} S^{*}\right)$-Ethyl $\quad 8^{\prime}$-bromo-2-oxo-3',8a'-dihydro-2' $H$ spiro[indoline-3,1'-indolizine]-3'-carboxylate (3f). Brown solid; $107 \mathrm{mg}$ (81\%); m.p. $144-146{ }^{\circ} \mathrm{C}$; IR $\left(\nu_{\max } / \mathrm{cm}^{-1}, \mathrm{CHCl}_{3}\right) 3434$, $2962,2361,1732,1621,1472,1374,1261,1106 ; \mathrm{NMR} \delta_{\mathrm{H}}$ $\left(400 \mathrm{MHz}, \mathrm{CDCl}_{3}\right) 8.55(1 \mathrm{H}, \mathrm{s}, \mathrm{NH}), 7.33-7.28(2 \mathrm{H}, \mathrm{m}, \operatorname{Ar} H)$, 7.09-7.04 (1H, m, ArH), 6.97 (1H, d, $J=7.3 \mathrm{~Hz}, \operatorname{Ar} H), 6.25(1 \mathrm{H}$, $\mathrm{d}, J=7.2 \mathrm{~Hz}, \mathrm{NCH}=\mathrm{CH}), 6.07(1 \mathrm{H}, \mathrm{d}, J=7.2 \mathrm{~Hz}, \mathrm{CH}=\mathrm{CBr})$, $5.38(1 \mathrm{H}, \mathrm{s}, \mathrm{CH}), 4.62(1 \mathrm{H}$, app.t, $J=7.2 \mathrm{~Hz}, \mathrm{NCH}=\mathrm{CH}), 4.44$ $\left(1 \mathrm{H}, \mathrm{dd}, J=8.4,7.9 \mathrm{~Hz}, \mathrm{CHCO}_{2} \mathrm{Et}\right), 4.29(2 \mathrm{H}, \mathrm{q}, J=7.1 \mathrm{~Hz}$, $\left.\mathrm{CH}_{2} \mathrm{CH}_{3}\right), 2.52\left(1 \mathrm{H}, \mathrm{dd}, J=13.4,7.9 \mathrm{~Hz}, \mathrm{CH}_{\mathrm{a}} \mathrm{H}_{\mathrm{b}}\right), 2.22(1 \mathrm{H}, \mathrm{dd}$, $\left.J=13.4,8.4 \mathrm{~Hz}, \mathrm{CH}_{\mathrm{a}} H_{\mathrm{b}}\right), 1.35\left(3 \mathrm{H}, \mathrm{t}, J=7.1 \mathrm{~Hz}, \mathrm{CH}_{2} \mathrm{CH}_{3}\right) ; \mathrm{NMR}$ $\delta_{\mathrm{C}}\left(100 \mathrm{MHz}, \mathrm{CDCl}_{3}\right) 178.0(\mathrm{C}), 171.7(\mathrm{C}), 141.1(\mathrm{C}), 132.8(\mathrm{CH})$, $130.6(\mathrm{C}), 128.6(\mathrm{CH}), 127.4(\mathrm{CH}), 123.6(\mathrm{CH}), 122.5(\mathrm{CH}), 110.0$ $(\mathrm{CH}), 109.3(\mathrm{C}), 94.9(\mathrm{CH}), 71.5(\mathrm{CH}), 64.1(\mathrm{CH}), 61.8\left(\mathrm{CH}_{2}\right)$, 60.9 (C), $37.7\left(\mathrm{CH}_{2}\right), 14.2\left(\mathrm{CH}_{3}\right) ; \mathrm{m} / z$ (HRMS-ESI+) $389.0494(\mathrm{M}$ $+\mathrm{H} \mathrm{C}_{18} \mathrm{H}_{18}{ }^{79} \mathrm{BrN}_{2} \mathrm{O}_{3}$ requires 389.0501), $391.0476(\mathrm{M}+\mathrm{H}$ $\mathrm{C}_{18} \mathrm{H}_{18}{ }^{81} \mathrm{BrN}_{2} \mathrm{O}_{3}$ requires 391.0480).

$\left(1^{\prime} S^{*}, 3^{\prime} R^{*}, 8 a^{\prime} S^{*}\right)$-Ethyl 6'-cyano-2-oxo-3',8a'-dihydro-2'H-spiro[indoline-3,1'-indolizine]-3'-carboxylate $(3 \mathrm{~g})$ and $\left(1^{\prime} R^{*}, 3^{\prime} R^{*}, 8 \mathrm{a}^{\prime}\right.$ $S^{*}$ )-ethyl $\quad 6^{\prime}$-cyano-2-oxo-3',8a'-dihydro-2' $H$-spiro[indoline-3,1'indolizine]-3'-carboxylate (39'). Yellow solid; $90 \mathrm{mg}$ (77\%); m.p. 188-190 ${ }^{\circ} \mathrm{C}$; IR $\left(\nu_{\max } / \mathrm{cm}^{-1}, \mathrm{CHCl}_{3}\right)$ 3203. 2981, 2938, $2203,1731,1643,1621,1574,1485,1469,1391,1133 ; \mathrm{NMR} \delta_{\mathrm{H}}$ (400 MHz, $d^{6}$-acetone, $2: 1$ mixture of diastereoisomers, *indicates minor where different from major) $9.65(1 \mathrm{H}$, br s, $\mathrm{NH})$, $9.50\left(1 \mathrm{H}\right.$, br s, $\left.\mathrm{NH}^{*}\right), 7.35-7.20(3 \mathrm{H}, \mathrm{m}, \mathrm{Ar} H, \mathrm{NCC}=\mathrm{C} H)$, 7.07-6.95 (2H, m, ArH), $5.80(1 \mathrm{H}$, app dt, $J=10.0,1.5 \mathrm{~Hz}$, $\left.\mathrm{CNC}-\mathrm{C} H^{*}=\mathrm{CH}\right), 5.67(1 \mathrm{H}$, app dt, $J=10.1,1.5 \mathrm{~Hz}, \mathrm{CNC}-$ $\mathrm{CH}=\mathrm{CH}), 5.06\left(1 \mathrm{H}, \mathrm{t}, J=2.1 \mathrm{~Hz}, \mathrm{NCH}^{*}\right), 4.98-4.93(2 \mathrm{H}, \mathrm{m}$, $\left.\mathrm{CHCO}_{2} \mathrm{Et}, \mathrm{NCH}\right), 4.86\left(1 \mathrm{H}\right.$, app t, $\left.J=7.7 \mathrm{~Hz}, \mathrm{CH}^{*} \mathrm{CO}_{2} \mathrm{Et}\right), 4.60$ $\left(1 \mathrm{H}, \mathrm{ddd}, J=10.0,2.1,1.0 \mathrm{~Hz}, \mathrm{CH}=\mathrm{CH}^{*}-\mathrm{CH}\right), 4.52(1 \mathrm{H}, \mathrm{ddd}$, $J=10.1,2.1,0.8 \mathrm{~Hz}, \mathrm{CH}=\mathrm{CH}-\mathrm{CH}), 4.35-4.24\left(2 \mathrm{H}, \mathrm{m}, \mathrm{CH}_{2} \mathrm{CH}_{3}\right)$, $2.57\left(1 \mathrm{H}, \mathrm{dd}, J=13.4,8.6 \mathrm{~Hz}, \mathrm{CH}_{\mathrm{a}}{ }^{*}\right), 2.48-2.43(2 \mathrm{H}, \mathrm{m}$, $\left.\mathrm{CH}_{2} \mathrm{CHCO}_{2} \mathrm{Et}\right), 2.37\left(1 \mathrm{H}, \mathrm{dd}, J=13.4,7.7 \mathrm{~Hz}, \mathrm{CH}_{b}{ }^{*}\right), 1.30(3 \mathrm{H}$, $\left.\mathrm{t}, J=7.1 \mathrm{~Hz}, \mathrm{CH}_{2} \mathrm{CH}_{3}\right)$; NMR $\delta_{\mathrm{C}}\left(100 \mathrm{MHz}, d^{6}\right.$-acetone) 179.3 (C), $176.5(\mathrm{C}), 171.8(\mathrm{C}), 171.6(\mathrm{C}), 147.8(\mathrm{CH}), 146.6(\mathrm{CH})$, 143.5 (C), 142.3 (C), $132.1(\mathrm{C}), 129.8(\mathrm{CH}), 129.4(\mathrm{CH}), 127.9$ (C), $126.4(\mathrm{C}), 124.7(\mathrm{CH}), 124.0(\mathrm{CH}), 123.7(\mathrm{CH}), 123.2(\mathrm{CH})$, 123.0 (CH), 120.9 (C), 120.7 (C) $111.9(\mathrm{CH}), 111.5(\mathrm{CH}), 110.7$ $(\mathrm{CH}), 110.2(\mathrm{CH}), 78.6(\mathrm{C}), 78.4(\mathrm{C}), 67.8(\mathrm{CH}), 67.4(\mathrm{CH}), 63.9$ 
$(\mathrm{CH}), 63.2(\mathrm{CH}), 62.4\left(\mathrm{CH}_{2}\right), 62.3\left(\mathrm{CH}_{2}\right), 37.2\left(\mathrm{CH}_{2}\right), 34.9\left(\mathrm{CH}_{2}\right)$, $14.5\left(\mathrm{CH}_{3}\right) ; m / z$ (HRMS-ESI+) $358.1162\left(\mathrm{M}+\mathrm{Na} \mathrm{C}_{19} \mathrm{H}_{17} \mathrm{~N}_{3} \mathrm{NaO}_{3}\right.$ requires 358.1162$)$.

$\left(1^{\prime} R^{*}, 3^{\prime} S^{*}, 8 a^{\prime} R^{*}\right)$-Ethyl 6'-carbamoyl-2-oxo-3',8a'-dihydro-2' $H$ spiro[indoline-3,1'-indolizine]-3'-carboxylate $(3 \mathrm{~h})$ and $\left(1^{\prime} R^{*}, 3^{\prime}\right.$ $S^{*}, 8 \mathrm{a}^{\prime} S^{*}$ )-ethyl $\quad 6^{\prime}$-carbamoyl-2-oxo-3',8a'-dihydro-2' $H$-spiro[indoline-3,1'-indolizine]-3'-carboxylate (3h'). Yellow solid; $109 \mathrm{mg}$ (95\%); m.p. $138-140{ }^{\circ} \mathrm{C}$; IR $\left(\nu_{\max } / \mathrm{cm}^{-1}, \mathrm{CHCl}_{3}\right) 3352$. 2926, 2359, 2340, 1710, 1648, 1620, 1585, 1471, 1382, 1290, 1196, 1104; NMR $\delta_{\mathrm{H}}\left(400 \mathrm{MHz}, d^{6}\right.$-DMSO, $7: 3$ mixture of diastereoisomers, *indicates minor where different from major) $10.63(1 \mathrm{H}, \mathrm{s}, \mathrm{NH}), 10.40\left(1 \mathrm{H}, \mathrm{s}, \mathrm{N} H^{*}\right), 7.38(2 \mathrm{H}, \mathrm{s}$, $\left.\mathrm{CH}=\mathrm{CCONH}_{2}\right), 7.27-7.16(4 \mathrm{H}, \mathrm{m}, \mathrm{Ar} H), 7.02-6.84(4 \mathrm{H}, \mathrm{m}$, $\operatorname{Ar} H), 6.54\left(4 \mathrm{H}\right.$, br s, $\left.\mathrm{NH}_{2}\right), 6.28(1 \mathrm{H}, \mathrm{dt}, J=10.2,2.1 \mathrm{~Hz}$, $\left.\mathrm{NH}_{2} \mathrm{COC}-\mathrm{CH}{ }^{*}=\mathrm{CH}\right), 6.14\left(1 \mathrm{H}, \mathrm{dt}, J=10.2,2.1 \mathrm{~Hz}, \mathrm{NH}_{2} \mathrm{COC}-\right.$ $\mathrm{CH}=\mathrm{CH}), 4.95\left(1 \mathrm{H}, \mathrm{dd}, J=8.8,7.4 \mathrm{~Hz}, \mathrm{CHCO}_{2} \mathrm{Et}\right), 4.92(1 \mathrm{H}$, $\left.\mathrm{dd}, J=8.8,7.4 \mathrm{~Hz}, \mathrm{CH}^{*} \mathrm{CO}_{2} \mathrm{Et}\right), 4.78(2 \mathrm{H}$, app dd, $J=2.1,0.6$ $\mathrm{Hz}, \mathrm{NCH}), 4.38\left(1 \mathrm{H}, \mathrm{ddd}, J=10.2,2.1,0.6 \mathrm{~Hz}, \mathrm{CH}=\mathrm{CH} H^{*} \mathrm{CH}\right)$, $4.35(1 \mathrm{H}, \mathrm{ddd}, J=10.2,2.1,0.6 \mathrm{~Hz}, \mathrm{CH}=\mathrm{CH}-\mathrm{CH}), 4.24(4 \mathrm{H}, \mathrm{m}$, $\left.\mathrm{CH}_{2} \mathrm{CH}_{3}\right), 2.47\left(1 \mathrm{H}, \mathrm{dd}, J=13.3,8.8 \mathrm{~Hz}, \mathrm{CH}_{\mathrm{a}}{ }^{*} \mathrm{H}_{\mathrm{b}}\right), 2.35-2.17$ $\left(2 \mathrm{H}, \mathrm{m}, \mathrm{CH}_{\mathrm{b}}{ }^{*}, \mathrm{CH}_{\mathrm{a}} H_{\mathrm{b}}\right), 1.29\left(3 \mathrm{H}, \mathrm{t}, J=7.1 \mathrm{~Hz}, \mathrm{CH}_{2} \mathrm{CH}_{3}{ }^{*}\right), 1.27$ $\left(3 \mathrm{H}, \mathrm{t}, J=7.1 \mathrm{~Hz}, \mathrm{CH}_{2} \mathrm{CH}_{3}\right)$; NMR $\delta_{\mathrm{C}}\left(100 \mathrm{MHz}, d^{6}\right.$-DMSO $)$ 179.3 (C), 176.6 (C), 172.2 (C), 172.1 (C), 167.4 (C), 167.3 (C), 143.0 (C), $142.6(\mathrm{CH}), 141.9(\mathrm{C}), 141.5(\mathrm{CH}), 132.0(\mathrm{C}) 129.0$ $(\mathrm{CH}), 128.7(\mathrm{CH}), 128.0(\mathrm{C}) 124.8(\mathrm{CH}), 124.2(\mathrm{CH}), 124.1(\mathrm{CH})$, $123.2(\mathrm{CH}), 122.1(\mathrm{CH}), 122.0(\mathrm{CH}) 110.0(\mathrm{CH}), 109.9(\mathrm{CH})$, $109.1(\mathrm{CH}), 107.5(\mathrm{CH}), 101.1(\mathrm{C}), 101.0(\mathrm{C}), 68.1(\mathrm{CH}), 67.5$ $(\mathrm{CH}), 63.5(\mathrm{CH}), 62.6(\mathrm{CH}), 61.8\left(\mathrm{CH}_{2}\right), 61.7\left(\mathrm{CH}_{2}\right), 55.6(\mathrm{C})$, $55.4(\mathrm{C}), 36.5\left(\mathrm{CH}_{2}\right), 34.3\left(\mathrm{CH}_{2}\right), 14.6\left(\mathrm{CH}_{3}\right), 14.5\left(\mathrm{CH}_{3}\right) ; \mathrm{m} / \mathrm{z}$ (HRMS-ESI+) $354.1442\left(\mathrm{M}+\mathrm{H} \mathrm{C}_{19} \mathrm{H}_{20} \mathrm{~N}_{3} \mathrm{O}_{4}\right.$ requires 354.1454).

$\left(1^{\prime} R^{*}, 3^{\prime} S^{*}, 8 \mathrm{a}^{\prime} R^{*}\right)$-Ethyl $6^{\prime}$-acetyl-2-oxo-3',8a'-dihydro-2'H-spiro[indoline-3, $1^{\prime}$-indolizine]-3'-carboxylate (3i) and $\left(1^{\prime} R^{*}, 3^{\prime} S^{*}, 8 a^{\prime}\right.$ $\left.S^{*}\right)$-ethyl $\quad 6^{\prime}$-acetyl-2-oxo-3',8a'-dihydro-2' $H$-spiro[indoline-3,1'indolizine]-3'-carboxylate (3i'). Brown solid, $97 \mathrm{mg}$ (81\%); m.p. $118-120{ }^{\circ} \mathrm{C}$; IR $\left(\nu_{\max } / \mathrm{cm}^{-1}, \mathrm{CHCl}_{3}\right) 3434,3010,2447,1731$, $1621,1573,1472,1373,1251,1180 ; \mathrm{NMR}_{\mathrm{H}}\left(400 \mathrm{MHz}, \mathrm{CDCl}_{3}\right.$, $1: 1.3$ mixture of diastereoisomers, *indicates minor where different from major) $8.35\left(1 \mathrm{H}, \mathrm{s}, \mathrm{N} H^{*}\right), 8.21(1 \mathrm{H}, \mathrm{s}, \mathrm{NH}), 7.47$ $\left(1 \mathrm{H}, \mathrm{s}, \quad \mathrm{NCH}^{*}=\mathrm{CCOCH}_{3}\right), 7.42\left(1 \mathrm{H}, \mathrm{s}, \mathrm{NCH}=\mathrm{CCOCH}_{3}\right)$, 7.27-6.94 (8H, m, ArH), $6.53\left(1 \mathrm{H}, \mathrm{d}, J=10.3 \mathrm{~Hz},\left(\mathrm{COCH}_{3}\right)\right.$ $\mathrm{CH}=\mathrm{CH}-\mathrm{CH}), 6.36\left(1 \mathrm{H}, \mathrm{d}, J=10.3 \mathrm{~Hz},\left(\mathrm{COCH}_{3}\right) \mathrm{CH}^{*}=\mathrm{CH}-\right.$ $\mathrm{CH}), 5.18\left(1 \mathrm{H}, \mathrm{t}, J=1.8 \mathrm{~Hz},\left(\mathrm{COCH}_{3}\right) \mathrm{CH}=\mathrm{CH}-\mathrm{CH}^{*}\right), 5.05(1 \mathrm{H}$, $\left.\mathrm{t}, J=1.8 \mathrm{~Hz},\left(\mathrm{COCH}_{3}\right) \mathrm{CH}=\mathrm{CH}-\mathrm{CH}\right), 4.81(1 \mathrm{H}, \mathrm{t}, J=7.8 \mathrm{~Hz}$, $\left.\mathrm{CHCO}_{2} \mathrm{Et}\right), 4.67\left(1 \mathrm{H}, \mathrm{dd}, J=10.3,1.8 \mathrm{~Hz},\left(\mathrm{COCH}_{3}\right) \mathrm{CH}=\mathrm{CH}-\right.$ $\mathrm{CH}) 4.61\left(1 \mathrm{H}, \mathrm{t}, J=7.8 \mathrm{~Hz}, \mathrm{CH}^{*} \mathrm{CO}_{2} \mathrm{Et}\right) 4.53(1 \mathrm{H}, \mathrm{dd}, J=10.3$, $\left.1.8 \mathrm{~Hz},\left(\mathrm{COCH}_{3}\right) \mathrm{CH}=\mathrm{CH}^{*}-\mathrm{CH}\right), 4.38-4.30\left(4 \mathrm{H}, \mathrm{m}, \mathrm{CH}_{2} \mathrm{CH}_{3}\right)$, 2.70-2.58 (2H, m, CH- $\left.\mathrm{CH}_{\mathrm{a}} \mathrm{CH}_{\mathrm{b}}\right), 2.45-2.33(2 \mathrm{H}, \mathrm{m}, \mathrm{CH}-$ $\left.\mathrm{CH}_{\mathrm{a}} \mathrm{CH}_{\mathrm{b}}\right), 2.18\left(3 \mathrm{H}, \mathrm{s}, \mathrm{COCH}_{3}{ }^{*}\right), 2.13\left(3 \mathrm{H}, \mathrm{s}, \mathrm{COCH}_{3}\right), 1.41-1.34$ $\left(3 \mathrm{H}, \mathrm{m}, \mathrm{CH}_{2} \mathrm{CH}_{3}\right)$; NMR $\delta_{\mathrm{C}}\left(100 \mathrm{MHz}, \mathrm{CDCl}_{3}\right) 191.8(\mathrm{C}), 179.6$ (C), 176.8 (C), $171.2(\mathrm{C}), 170.6(\mathrm{C}), 146.2(\mathrm{CH}), 144.3(\mathrm{CH})$, 141.6 (C), 140.3 (C), 130.7 (C), $129.2(\mathrm{CH}), 128.9(\mathrm{CH}), 126.8$ (C), $123.9(\mathrm{CH}), 123.7(\mathrm{CH}), 123.5(\mathrm{CH}), 123.2(\mathrm{CH}), 122.9(\mathrm{CH})$, $122.8(\mathrm{CH}), 122.6(\mathrm{CH}), 110.4(\mathrm{CH}), 110.3(\mathrm{CH}), 108.7(\mathrm{CH})$, $68.1(\mathrm{CH}), 67.1(\mathrm{CH}), 63.3(\mathrm{CH}), 62.8(\mathrm{CH}), 62.4\left(\mathrm{CH}_{2}\right), 62.2$ $\left(\mathrm{CH}_{2}\right), 58.8(\mathrm{C}), 58.4(\mathrm{C}), 36.9\left(\mathrm{CH}_{2}\right), 34.4\left(\mathrm{CH}_{2}\right), 14.2\left(\mathrm{CH}_{3}\right) ; \mathrm{m} / \mathrm{z}$ (HRMS-ESI +$) 353.1498\left(\mathrm{M}+\mathrm{H} \mathrm{C}_{20} \mathrm{H}_{21} \mathrm{~N}_{2} \mathrm{O}_{4}\right.$ requires 353.1501). $\left(1^{\prime} R^{*}, 3^{\prime} S^{*}, 8 a^{\prime} S^{*}\right)-3^{\prime}$-Acetyl-8'-bromo-3',8a'-dihydro-2'H-spiro [indoline-3,1'-indolizin]-2-one (3j). Brown solid; $63 \mathrm{mg}$ (76\%); m.p. 126-128 ${ }^{\circ} \mathrm{C}$; IR $\left(\nu_{\max } / \mathrm{cm}^{-1}, \mathrm{CHCl}_{3}\right)$ 3438, 3011, 2928, 2437, 1780, 1721, 1622, 1471, 1426, 1390, 1240, 1170; NMR $\delta_{\mathrm{H}}\left(400 \mathrm{MHz}, d^{6}\right.$-DMSO) $10.53(1 \mathrm{H}, \mathrm{s}, \mathrm{NH}), 7.27-7.19(2 \mathrm{H}$, m, $2 \times \operatorname{ArH}), 6.96(1 \mathrm{H}, \mathrm{td}, J=7.6,1.0 \mathrm{~Hz}, \operatorname{Ar} H), 6.86(1 \mathrm{H}, \mathrm{d}, J=$ $7.6 \mathrm{~Hz}, \mathrm{ArH}), 6.43(1 \mathrm{H}, \mathrm{d}, J=7.0 \mathrm{~Hz}, \mathrm{CH}-\mathrm{CH}=\mathrm{CBr}), 6.09(1 \mathrm{H}$, $\mathrm{d}, J=7.0 \mathrm{~Hz}, \mathrm{~N}-\mathrm{CH}=\mathrm{CH}), 4.88(1 \mathrm{H}, \mathrm{s}, \mathrm{NCH}), 4.70(1 \mathrm{H}, \mathrm{t}, J=7.0$ $\mathrm{Hz}, \mathrm{NCH}=\mathrm{CH}), 4.49\left(1 \mathrm{H}, \mathrm{t}, J=6.8 \mathrm{~Hz}, \mathrm{CHCOCH}_{3}\right), 2.19(3 \mathrm{H}, \mathrm{s}$, $\left.\mathrm{COCH}_{3}\right), 2.11-2.06\left(2 \mathrm{H}, \mathrm{m}, \mathrm{CH}_{\mathrm{a}} H_{\mathrm{b}}\right)$; NMR $\delta_{\mathrm{C}}\left(100 \mathrm{MHz}, d^{6}-\right.$ DMSO) 207.3 (C), 177.7 (C), 142.8 (C), 135.2 (CH), 131.3 (C), $128.7(\mathrm{CH}), 128.2(\mathrm{CH}), 123.8(\mathrm{CH}), 121.8(\mathrm{CH}), 109.9(\mathrm{CH})$, $108.0(\mathrm{C}), 93.3(\mathrm{CH}), 71.7(\mathrm{CH}), 70.5(\mathrm{CH}), 61.3(\mathrm{C}), 36.4\left(\mathrm{CH}_{2}\right)$, $26.7\left(\mathrm{CH}_{3}\right) ; \mathrm{m} / z$ (HRMS-ESI+) $359.0370\left(\mathrm{M}+\mathrm{H} \mathrm{C}_{17} \mathrm{H}_{16}{ }^{79} \mathrm{BrN}_{2} \mathrm{O}_{2}\right.$ requires 359.0395), $361.0359\left(\mathrm{M}+\mathrm{H} \mathrm{C}_{17} \mathrm{H}_{16}{ }^{81} \mathrm{BrN}_{2} \mathrm{O}_{2}\right.$ requires $361.0375)$.

$\left(2^{\prime} S^{*}, 3^{\prime} S^{*}, 8 a^{\prime} S^{*}\right)$-Diethyl $\quad 8^{\prime}$-fluoro-2-oxo-3',8a'-dihydro-2' $H^{-}$ spiro[indoline-3,1'-indolizine]-2', $\mathbf{3}^{\prime}$-dicarboxylate (3k). Yellow oil; $80 \mathrm{mg}$ (87\%); IR $\left(\nu_{\max } / \mathrm{cm}^{-1}, \mathrm{CHCl}_{3}\right)$ 3691, 3440, 3010, $1736,1620,1597,1472,1391,1314,1192,1025 ; \mathrm{NMR} \delta_{\mathrm{H}}$ (400 MHz, d $d^{6}$-acetone) $9.66(1 \mathrm{H}, \mathrm{br} \mathrm{s}, \mathrm{NH}), 7.26-7.22(2 \mathrm{H}, \mathrm{m}$, $\mathrm{Ar} H)$, 6.97-6.91 (2H, m, $\mathrm{ArH}), 6.35-6.32(1 \mathrm{H}, \mathrm{m}, \mathrm{N}-\mathrm{CH}=\mathrm{CH})$, 5.39-5.34 (1H, m, CH-CH=CF), $5.17(1 \mathrm{H}, \mathrm{s}, \mathrm{NCHC}-\mathrm{F}), 5.03$ $\left(1 \mathrm{H}, \mathrm{dd}, J=7.4,1.2 \mathrm{~Hz}, \mathrm{NCH}\left(\mathrm{CO}_{2} \mathrm{Et}\right)\right), 4.54-4.49(1 \mathrm{H}, \mathrm{m}$, $\mathrm{NCH}=\mathrm{CH}), 4.27\left(2 \mathrm{H}, \mathrm{q}, J=7.1 \mathrm{~Hz}, \mathrm{NCHCO}_{2} \mathrm{CH}_{2} \mathrm{CH}_{3}\right)$, 3.81-3.66 (3H, m, $\mathrm{NCH}\left(\mathrm{CO}_{2} \mathrm{Et}\right) \mathrm{CH}\left(\mathrm{CO}_{2} \mathrm{Et}\right), \mathrm{NCH}\left(\mathrm{CO}_{2} \mathrm{Et}\right) \mathrm{CH}-$ $\left.\left(\mathrm{CO}_{2} \mathrm{CH}_{2} \mathrm{CH}_{3}\right)\right), 1.29\left(3 \mathrm{H}, \mathrm{t}, J=7.1 \mathrm{~Hz}, \mathrm{NCHCOCH}_{2} \mathrm{CH}_{3}\right), 0.70$ $\left(3 \mathrm{H}, \mathrm{t}, J=7.1 \mathrm{~Hz}, \mathrm{NCH}\left(\mathrm{CO}_{2} \mathrm{Et}\right) \mathrm{CH}\left(\mathrm{CO}_{2} \mathrm{CH}_{2} \mathrm{CH}_{3}\right)\right) ; \mathrm{NMR} \delta_{\mathrm{C}}$ (100 MHz, d $d^{6}$-acetone) 174.9 (C), 170.9 (C), 168.1 (C), 149.3 (d, $\left.J^{C-F}=252 \mathrm{~Hz}, \mathrm{C}\right), 143.0(\mathrm{C}), 130.5\left(\mathrm{~d}, J^{C-F}=4 \mathrm{~Hz}, \mathrm{CH}\right), 129.0$ $(\mathrm{CH}), 126.6(\mathrm{C}), 124.6(\mathrm{CH}), 121.3(\mathrm{CH}), 109.6(\mathrm{CH}), 103.1(\mathrm{~d}$, $\left.J^{C-F}=14 \mathrm{~Hz}, \mathrm{CH}\right), 91.1\left(\mathrm{~d}, J^{C-F}=5 \mathrm{~Hz}, \mathrm{CH}\right), 68.0\left(\mathrm{~d}, J^{C-F}=33\right.$ $\mathrm{Hz}, \mathrm{CH}), 65.3\left(\mathrm{~d}, J^{C-F}=1 \mathrm{~Hz}, \mathrm{CH}\right), 62.6\left(\mathrm{~d}, J^{C-F}=3 \mathrm{~Hz}, \mathrm{C}\right), 61.5$ $\left(\mathrm{CH}_{2}\right), 60.9\left(\mathrm{CH}_{2}\right), 52.9(\mathrm{CH}), 13.6\left(\mathrm{CH}_{3}\right), 12.8\left(\mathrm{CH}_{3}\right) ; \mathrm{m} / \mathrm{z}$ (HRMS-ESI +$) \quad 401.1507\left(\mathrm{M}+\mathrm{H} \quad \mathrm{C}_{21} \mathrm{H}_{22} \mathrm{FN}_{2} \mathrm{O}_{5}\right.$ requires 401.1507).

$\left(1^{\prime} R^{*}, 2^{\prime} S^{*}, 3^{\prime} S^{*}, 8 a^{\prime} R^{*}\right)$-Diethyl 2-oxo-3', $5^{\prime}, 6^{\prime}, 7^{\prime}, 8^{\prime}, 8 a^{\prime}-$ hexahydro$2^{\prime} H$-spiro[indoline-3, $1^{\prime}$-indolizine]-2', $3^{\prime}$-dicarboxylate (31). Following the general procedure described above but the product was not stable to chromatography on silica gel, so the crude cycloadduct $\mathbf{4}$ was reduced without purification using the following procedure.

Crude cycloadduct 4 (75 mg) and RANEY® nickel (10 mol\%) were dissolved in anhydrous ethyl acetate $(4 \mathrm{~mL})$, under argon. The atmosphere was replaced with $\mathrm{H}_{2}$ and the mixture stirred until reduction was complete by TLC analysis and then filtered through a pad of Celite. The filtrate was concentrated in vacuo and purified via column chromatography (EtOAcpetrol; $1 / 4)$ to give spiroindolizidine 31 as a yellow oil; $48 \mathrm{mg}$ (63\%); IR $\left(\nu_{\max } / \mathrm{cm}^{-1}, \mathrm{CHCl}_{3}\right) 3440,3198,2942,1732,1621$, $1471,1373,1339,1319,1184,1097$; NMR $\delta_{\mathrm{H}}\left(400 \mathrm{MHz}, \mathrm{CDCl}_{3}\right)$ $8.66(1 \mathrm{H}$, br s, NH), $7.31(1 \mathrm{H}, \mathrm{d}, J=7.6 \mathrm{~Hz}, \operatorname{Ar} H), 7.22(1 \mathrm{H}, \mathrm{td}$, $J=7.6,1.0 \mathrm{~Hz}, \operatorname{Ar} H), 7.00(1 \mathrm{H}, \mathrm{t}, J=7.6 \mathrm{~Hz}, \operatorname{Ar} H), 6.92(1 \mathrm{H}, \mathrm{d}, J=$ $7.6 \mathrm{~Hz}, \mathrm{ArH}), 4.59\left(1 \mathrm{H}, \mathrm{d}, J=5.6 \mathrm{~Hz}, \mathrm{NCHCO}_{2} \mathrm{Et}\right), 4.35-4.20(2 \mathrm{H}$, $\left.\mathrm{m}, \mathrm{NCH}\left(\mathrm{COCH}_{2} \mathrm{CH}_{3}\right)\right), 4.00\left(1 \mathrm{H}, \mathrm{d}, J=5.6 \mathrm{~Hz}, \mathrm{NCH}\left(\mathrm{CO}_{2} \mathrm{Et}\right) \mathrm{CH}\right)$, 3.81-3.61 (2H, m, $\left.\mathrm{CH}\left(\mathrm{COCH}_{2} \mathrm{CH}_{3}\right)\right), 3.41$ (1H, dd, $J=10.9,2.4$ 
$\mathrm{Hz}, \mathrm{NCHC})$ ), $3.09\left(1 \mathrm{H}, \mathrm{d}, J=9.5 \mathrm{~Hz}, \mathrm{CH}_{\mathrm{a}} \mathrm{H}_{\mathrm{b}}\right), 2.45(1 \mathrm{H}, \mathrm{t}, J=9.5$ $\left.\mathrm{Hz}, \mathrm{CH}_{\mathrm{a}} H_{\mathrm{b}}\right), 1.64-1.53\left(2 \mathrm{H}, \mathrm{m}, \mathrm{CH}_{\mathrm{a}} \mathrm{H}_{\mathrm{b}}, \mathrm{CH}_{\mathrm{a}} H_{\mathrm{b}}\right), 1.42-1.12(6 \mathrm{H}$, $\left.\mathrm{m}, \mathrm{CH}_{\mathrm{a}} \mathrm{H}_{\mathrm{b}}, \mathrm{CH}_{\mathrm{a}} \mathrm{H}_{\mathrm{b}}, \mathrm{CH}_{\mathrm{a}} \mathrm{CH}_{\mathrm{b}}, \mathrm{CH}_{2} \mathrm{CH}_{3}\right), 0.74-0.64(4 \mathrm{H}, \mathrm{m}$, $\left.\mathrm{CH}_{2} \mathrm{CH}_{3}, \mathrm{CH}_{\mathrm{a}} \mathrm{CH}_{\mathrm{b}}\right) ; \mathrm{NMR} \delta_{\mathrm{C}}\left(100 \mathrm{MHz}, \mathrm{CDCl}_{3}\right) 178.4(\mathrm{C}), 172.4$ (C), 143.1 (C), 131.7 (C), 128.2 (CH), 126.5 (C), $125.8(\mathrm{CH}), 122.2$ $(\mathrm{CH}), 109.4(\mathrm{CH}), 67.7(\mathrm{CH}), 64.1(\mathrm{CH}), 60.9\left(\mathrm{CH}_{2}\right), 60.6\left(\mathrm{CH}_{2}\right)$, $60.0(\mathrm{C}), 54.2(\mathrm{CH}), 47.5\left(\mathrm{CH}_{2}\right), 25.9\left(\mathrm{CH}_{2}\right), 25.3\left(\mathrm{CH}_{2}\right), 22.6$ $\left(\mathrm{CH}_{2}\right), 14.4\left(\mathrm{CH}_{3}\right), 13.3\left(\mathrm{CH}_{3}\right) ; m / z$ (HRMS-ESI+) $387.1908(\mathrm{M}+\mathrm{H}$ $\mathrm{C}_{21} \mathrm{H}_{27} \mathrm{~N}_{2} \mathrm{O}_{5}$ requires 387.1920).

$\left(1^{\prime} S^{*}, 2^{\prime} R^{*}, 3^{\prime} R^{*}, 8 \mathrm{a}^{\prime} R^{*}\right)$-Ethyl 8'-bromo-3'-cyano-2-oxo-3',8a'-dihydro$\mathbf{2}^{\prime} \boldsymbol{H}$-spiro[indoline-3,1'-indolizine]-2'-carboxylate (3m). White solid; $145 \mathrm{mg}$ (72\%); m.p. 159-161 ${ }^{\circ} \mathrm{C}$ (acetone); IR $\left(\nu_{\max } / \mathrm{cm}^{-1}\right.$, $\mathrm{CHCl}_{3}$ ) 3691, 3439, 3009, 2482, 2245, 1735, 1640, 1621, 1569, 1485, 1378, 1191; NMR $\delta_{\mathrm{H}}\left(400 \mathrm{MHz}, d^{6}\right.$-acetone) $9.74(1 \mathrm{H}, \mathrm{br}$ s, NH), $7.28(1 \mathrm{H}$, app t, $J=7.6 \mathrm{~Hz}, \operatorname{Ar} H), 7.22(1 \mathrm{H}, \mathrm{d}, J=7.3 \mathrm{~Hz}$, $\operatorname{Ar} H)$, 7.01-6.91 (2H, m, $\operatorname{Ar} H), 6.67(1 \mathrm{H}, \mathrm{d}, J=7.2, \mathrm{~N}-\mathrm{CH}=\mathrm{CH})$, $6.12(1 \mathrm{H}, \mathrm{d}, J=6.4 \mathrm{~Hz}, \mathrm{CH}-\mathrm{CH}=\mathrm{CBr}), 5.48(1 \mathrm{H}, \mathrm{d}, J=6.4 \mathrm{~Hz}$, NCHCN, 5.25 (1H, s, NCHC-Br), $4.72(1 \mathrm{H}$, app t, $J=6.4 \mathrm{~Hz}$, $\mathrm{NCH}=\mathrm{CH}), 3.77\left(1 \mathrm{H}, \mathrm{d}, J=6.4 \mathrm{~Hz}, \mathrm{CHCO}_{2} \mathrm{CH}_{2} \mathrm{CH}_{3}\right), 3.72(2 \mathrm{H}$, $\left.\mathrm{q}, J=7.1 \mathrm{~Hz}, \mathrm{CCH}\left(\mathrm{CO}_{2} \mathrm{CH}_{2} \mathrm{CH}_{3}\right)\right), 0.74(3 \mathrm{H}, \mathrm{t}, J=7.1 \mathrm{~Hz}$, $\left.\mathrm{NCHCOCH}_{2} \mathrm{CH}_{3}\right)$; NMR $\delta_{\mathrm{C}}\left(100 \mathrm{MHz}, d^{6}\right.$-DMSO) 176.1 (C), $167.9(\mathrm{C}), 144.6(\mathrm{C}), 132.8(\mathrm{CH}), 130.3(\mathrm{CH}), 129.1(\mathrm{CH}), 126.7$ (C), $125.5(\mathrm{CH}), 122.3(\mathrm{CH}), 119.1$ (C), $110.6(\mathrm{CH}), 109.1$ (C), $96.7(\mathrm{CH}), 72.9(\mathrm{CH}), 63.8(\mathrm{C}), 62.3\left(\mathrm{CH}_{2}\right), 55.6(\mathrm{CH}), 54.9$ (CH), $13.7\left(\mathrm{CH}_{3}\right) ; \mathrm{m} / \mathrm{z}$ (HRMS-ESI+) $436.0273(\mathrm{M}+\mathrm{Na}$ $\mathrm{C}_{19} \mathrm{H}_{16}{ }^{79} \mathrm{BrN}_{3} \mathrm{NaO}_{3}$ requires 436.0273), 438.0256 ( $\mathrm{M}+\mathrm{Na}$ $\mathrm{C}_{19} \mathrm{H}_{16}{ }^{81} \mathrm{BrN}_{3} \mathrm{NaO}_{3}$ requires 438.0256).

$\left(1^{\prime} S^{*}, 2^{\prime} R^{*}, 3^{\prime} R^{*}, 8 a^{\prime} R^{*}\right)$-Ethyl 8'-bromo-2-oxo-3'-phenyl-3',8a'-dihydro$\mathbf{2}^{\prime} \boldsymbol{H}$-spiro[indoline-3,1'-indolizine]-2'-carboxylate (3n). White solid; $149 \mathrm{mg}$ (70\%); m.p. 176-178 ${ }^{\circ} \mathrm{C}$ (acetone); IR $\left(\nu_{\max } / \mathrm{cm}^{-1}\right.$, $\mathrm{CHCl}_{3}$ ) 3692, 3441, 3009, 1731, 1601, 1562, 1484, 1379, 1247, 1117, 1027; NMR $\delta_{\mathrm{H}}\left(400 \mathrm{MHz}, d^{6}\right.$-acetone) $9.71(1 \mathrm{H}, \mathrm{br} \mathrm{s}, \mathrm{NH})$, 7.51-7.41 (5H, m, ArH), 7.36-7.25 (2H, m, ArH), $7.01(1 \mathrm{H}, \mathrm{td}$, $J=7.7,0.8 \mathrm{~Hz}, \operatorname{Ar} H), 6.97(1 \mathrm{H}, \mathrm{d}, J=7.7 \mathrm{~Hz}, \operatorname{Ar} H), 6.32(1 \mathrm{H}, \mathrm{d}, J=$ $6.7 \mathrm{~Hz}, \mathrm{~N}-\mathrm{CH}=\mathrm{CH}), 6.07(1 \mathrm{H}, \mathrm{d}, J=6.7 \mathrm{~Hz}, \mathrm{CH}-\mathrm{CH}=\mathrm{CBr}), 5.43$ $(1 \mathrm{H}, \mathrm{d}, J=8.5 \mathrm{~Hz}, \mathrm{NCHPh}, 4.51(1 \mathrm{H}$, app t, $J=6.7 \mathrm{~Hz}$, $\mathrm{C} H=\mathrm{CHN}), 3.78\left(1 \mathrm{H}, \mathrm{dq}, J=10.8,7.1 \mathrm{~Hz}, \mathrm{CH}_{a} \mathrm{H}_{\mathrm{b}}\right), 3.67(1 \mathrm{H}, \mathrm{dq}$, $\left.J=10.8,7.1 \mathrm{~Hz}, \mathrm{CH}_{\mathrm{a}} H_{b}\right), 3.4\left(1 \mathrm{H}, \mathrm{d}, J=8.5 \mathrm{~Hz}, \mathrm{CHCO}_{2} \mathrm{Et}\right), 0.77$ $\left(3 \mathrm{H}, \mathrm{t}, J=7.1 \mathrm{~Hz}, \mathrm{CH}_{3}\right)$; NMR $\delta_{\mathrm{C}}\left(100 \mathrm{MHz}, d^{6}\right.$-acetone $) 176.6(\mathrm{C})$, 169.3 (C), 144.5 (C), 143.2 (C), $134.6(\mathrm{CH}), 130.0(\mathrm{CH}), 129.9$ $(\mathrm{CH}), 129.4(\mathrm{CH}), 128.7(\mathrm{CH}), 127.5(\mathrm{C}), 127.2(\mathrm{CH}), 125.6(\mathrm{CH})$, $122.2(\mathrm{CH}), 110.4(\mathrm{CH}), 108.3(\mathrm{C}), 94.1(\mathrm{CH}), 73.7(\mathrm{CH})$, $68.1(\mathrm{CH}), 64.8(\mathrm{C}), 61.6\left(\mathrm{CH}_{2}\right), \quad 59.4(\mathrm{CH}), 13.8\left(\mathrm{CH}_{3}\right)$; $m / z \quad$ (HRMS-ESI + ) $465.0800\left(\mathrm{M}+\mathrm{H} \quad \mathrm{C}_{24} \mathrm{H}_{22}{ }^{79} \mathrm{BrN}_{2} \mathrm{O}_{3}\right.$ requires 465.0808), $467.0786\left(\mathrm{M}+\mathrm{H} \mathrm{C}_{24} \mathrm{H}_{22}{ }^{81} \mathrm{BrN}_{2} \mathrm{O}_{3}\right.$ requires 465.0793).

Diethyl 2-(2-oxoindolin-3-ylidene)succinate 5. Method as cycloaddition, but ethanol was used as solvent and reaction performed at $50{ }^{\circ} \mathrm{C}$. Yellow oil (60\%); NMR $\delta_{\mathrm{H}}(400 \mathrm{MHz}$, $\mathrm{CDCl}_{3}, 5: 1$ mixture of diastereoisomers, only major diastereoisomer peaks are quoted) $\left(\nu_{\max } / \mathrm{cm}^{-1}, \mathrm{CHCl}_{3}\right) 3446,3011,2986$, $1726,1617,1470,1370,1334,1295,1192$; NMR $\delta_{\mathrm{H}}(400 \mathrm{MHz}$, $\left.\mathrm{CDCl}_{3}\right) 8.38(1 \mathrm{H}, \mathrm{s}, \mathrm{NH}), 7.66(1 \mathrm{H}, \mathrm{d}, J=8.0 \mathrm{~Hz}, \mathrm{Ar} H), 7.29(1 \mathrm{H}$, $\mathrm{td}, J=8.0,1.2 \mathrm{~Hz}, \operatorname{Ar} H), 6.98(1 \mathrm{H}, \mathrm{td}, J=8.0,1.2 \mathrm{~Hz}, \operatorname{Ar} H), 6.85$ $(1 \mathrm{H}, \mathrm{d}, J=8.0 \mathrm{~Hz}, \mathrm{ArH}), 4.43\left(2 \mathrm{H}, \mathrm{q}, J=7.2 \mathrm{~Hz}, \mathrm{CH}_{2} \mathrm{CH}_{3}\right), 4.41$ $\left(2 \mathrm{H}, \mathrm{s}, \mathrm{CH}_{2} \mathrm{CO}_{2} \mathrm{Et}\right), 4.22\left(2 \mathrm{H}, \mathrm{q}, J=7.2 \mathrm{~Hz}, \mathrm{CH}_{2} \mathrm{CH}_{3}\right), 1.40(3 \mathrm{H}$, $\left.\mathrm{t}, J=7.2 \mathrm{~Hz}, \mathrm{CH}_{2} \mathrm{CH}_{3}\right), 1.29\left(3 \mathrm{H}, \mathrm{t}, J=7.2 \mathrm{~Hz}, \mathrm{CH}_{2} \mathrm{CH}_{3}\right) ; \mathrm{NMR}$ $\delta_{\mathrm{C}}\left(100 \mathrm{MHz}, \mathrm{CDCl}_{3}\right) 169.7$ (C), 169.2 (C), 167.4 (C), 141.0 (C), 135.0 (C), $130.9(\mathrm{CH}), 129.4(\mathrm{C}), 125.1(\mathrm{CH}), 122.3(\mathrm{CH}), 120.8$ (C), $109.9(\mathrm{CH}), 61.9\left(\mathrm{CH}_{2}\right), 61.2\left(\mathrm{CH}_{2}\right), 34.7\left(\mathrm{CH}_{2}\right), 14.2\left(\mathrm{CH}_{3}\right)$, $14.0\left(\mathrm{CH}_{3}\right) ; \mathrm{m} / \mathrm{z}$ (HRMS-ESI+) $326.0982([\mathrm{M}+\mathrm{Na}] 100 \%)$ requires $\mathrm{C}_{16} \mathrm{H}_{17} \mathrm{NO}_{5} \mathrm{Na}^{+} 326.0999$.

\section{Acknowledgements}

This work was supported by the EPSRC (MU, EP/J021008/1), AstraZeneca (JD) and the University of Nottingham (RAC).

\section{Notes and references}

1 B. Over, S. Wetzel, C. Grutter, Y. Nakai, S. Renner, D. Rauh and H. Waldmann, Nat. Chem., 2013, 5, 21-28.

2 C. V. Galliford and K. A. Scheidt, Angew. Chem., Int. Ed., 2007, 46, 8748-8758.

3 C. Marti and E. M. Carreira, Eur. J. Org. Chem., 2003, 22092219.

4 G. S. Singh and Z. Y. Desta, Chem. Rev., 2012, 112, 61046155.

5 For recent examples and references: (a) A. P. Antonchick, C. Gerding-Reimers, M. Catarinella, M. Schürmann, H. Preut, S. Ziegler, D. Rauh and H. Waldmann, Nat. Chem., 2010, 2, 735-740; (b) T.-L. Liu, Z.-Y. Xue, H.-Y. Tao and C.-J. Wang, Org. Biomol. Chem., 2011, 9, 1980-1986.

6 A. B. Serov, V. G. Kartsev, Y. A. Aleksandrov and F. M. Dolgushin, Russ. Chem. Bull., 2005, 54, 2432-2436.

7 L. Wu, J. Sun and C.-G. Yan, Org. Biomol. Chem., 2012, 10, 9452-9463.

8 B. X. Wang, X. C. Zhang, J. Li, X. Jiang, Y. F. Hu and H. W. Hu, J. Chem. Soc., Perkin Trans. 1, 1999, 1571-1575. See ref. 22 also.

9 S. Kanemasa, S. Takenaka, H. Watanabe and O. Tsuge, J. Org. Chem., 1989, 54, 420-424.

10 S. J. Taylor, A. M. Taylor and S. L. Schreiber, Angew. Chem., Int. Ed., 2004, 43, 1681-1685.

11 For recent developments: M. A. Ischay, M. K. Takase, R. G. Bergman and J. A. Ellman, J. Am. Chem. Soc., 2013, 135, 2478-2481 and references therein.

12 (a) For Wittig olefination for 1a-e: S. W. Duan, Y. Li, Y. Y. Liu, Y. Q. Zou, D. Q. Shi and W. J. Xiao, Chem. Commun., 2012, 48, 5160-5162; (b) For Peterson route to 1f: T. Watanabe, M. Arisawa, K. Narusuye, M. S. Alam, K. Yamamoto, M. Mitomi, Y. Ozoe and A. Nishida, Bioorg. Med. Chem., 2009, 17, 94-110.

13 A. R. Katritzky, N. E. Grzeskowiak and J. Alvarezbuilla, J. Chem. Soc., Perkin Trans. 1, 1981, 1180-1185.

14 W. G. Phillips and K. W. Ratts, J. Org. Chem., 1970, 35, 3144-3147.

15 X.-M. Zhang, F. G. Bordwell, M. Van Der Puy and H. E. Fried, J. Org. Chem., 1993, 58, 3060-3066. 
16 R. L. Hinman and C. P. Bauman, J. Org. Chem., 1964, 29, 2431-2437.

17 For a related synthetic process: B. Viswambharan, K. Selvakumar, S. Madhavan and P. Shanmugam, Org. Lett., 2010, 12, 2108-2111.

18 G. Laus, J. Chem. Soc., Perkin Trans. 2, 1998, 315-317.

19 Diastereoisomers, such as $\mathbf{3} \mathbf{g}: \mathbf{3} \mathbf{g}^{\prime}$ could not be separated by column chromatography on silica gel. No change was observed to the diastereomeric ratio in the crude product obtained from cycloaddition reactions performed in various solvents at a range of temperatures.

20 Q. Fu and C.-G. Yan, Tetrahedron, 2013, 69, 5841-5849.

21 For related solvent effects: N. E. Dontsova, V. N. Nesterov and A. M. Shestopalov, Tetrahedron, 2013, 69, 5016-5021.

22 A very recent paper describing stepwise reaction of pyridinium ylides with Michael acceptors: D. S. Allgäuer and H. Mayr, Eur. J. Org. Chem., 2013, DOI: 10.1002/ ejoc. 201300784. 